Int. J. Electrochem. Sci., 11 (2016) 9949 - 9958

\title{
A New Method for Puerarin Determination Based on Poly (Alizarin Red S/Graphene) Modified Electrode
}

\author{
Weili Zhang , Pingping Zhang, Hua Zhang, Jinyi Qin, Qinglu Wang \\ College of Pharmacy, Key Laboratory of Biomedical Engineering and Technology in Universities of \\ Shandong, Qilu Medical University, Zibo 255213, P. R. China. \\ *E-mail: zhangweili4619563@126.com
}

doi: $10.20964 / 2016.12 .60$

Received: 22 August 2016 / Accepted: 13 October 2016 / Published: 10 November 2016

\begin{abstract}
A poly(alizarin red S/graphene) (PARS-GR) composite membrane modified glassy carbon electrode was constructed by a facial one step electrodeposition method. The surface morphology and electrochemical characteric of the modified electrode were investigated carefully by the assistance of scanning electron microscopy and cyclic voltammetry, respectively. The electrochemical behavior of puerarin was investigated by cyclic voltammetry and a oxidation peak appeared at $+0.61 \mathrm{~V}$ (vs. SCE) in $\mathrm{pH} 6.5$ phosphate buffer solution. The PAR-GR/GCE displayed satisfactory electrochemical properties including higher conductivity and lower electron flowing resistance. The oxidation process of puerarin on PAR-GR/GCE surface is calculated to be a one-electron and one-proton transfer process. Under the optimized experimental conditions, the oxidation peak current showed good linearity with puerarin concentration under the range of $1.0 \times 10^{-7} \sim 7.5 \times 10^{-4} \mathrm{~mol} / \mathrm{L}$ accompanied by a detection limit of $3.4 \times 10^{-8} \mathrm{~mol} / \mathrm{L}(3 \sigma)$. The modified electrode was successfully utilized to examine the puerarin samples with satisfactory results.
\end{abstract}

Keywords: Puerarin, Alizarin red S, Graphene, Chemical modified electrode

\section{$\underline{\text { FULL TEXT }}$}

(C) 2016 The Authors. Published by ESG (www.electrochemsci.org). This article is an open access article distributed under the terms and conditions of the Creative Commons Attribution license (http://creativecommons.org/licenses/by/4.0/). 